Canad. Math. Bull. Vol. 23 (3) 1980

\title{
A NEW IDENTITY AND SOME APPLICATIONS
}

\author{
BY \\ W. O. J. MOSER ${ }^{1}$ AND RICHARD POLLACK ${ }^{2}$
}

\begin{abstract}
Let $(n \mid k)$ denote the number of $k$-choices $1 \leq x_{1}<$ $x_{2}<\cdots<x_{k} \leq n$ satisfying $x_{i}-x_{i-1} \geq 2, i=2, \ldots, k, n+x_{1}-x_{k} \geq 2$; let $(m, n \mid k)=\sum_{i+i=k}(m \mid i)(n \mid j)$. Several elementary proofs of the new identity $(m, n \mid k)=(m+n \mid k)$ if $0 \leq k<m \leq n$ and

$$
(m, n \mid k)=(m+n \mid k)+(-1)^{m}(n-m \mid k-m)
$$

if $0 \leq m \leq n, m \leq k$, are given. Generalizations and applications are considered.
\end{abstract}

1. For non-negative integers $n, k, w(n, k$ not both 0$)$, let $(n \mid k)_{w}$ denote the number of $k$-choices ( $k$-subsets)

$$
1 \leq x_{1}<x_{2}<\cdots<x_{k} \leq n
$$

satisfying the conditions

$$
x_{i}-x_{i-1} \geq w+1, \quad i=2, \ldots, k
$$

and

$$
x_{1}+n-x_{k} \geq w+1 .
$$

These conditions are best visualized by displaying $1,2, \ldots, n$ in a circle (rising order clockwise) and conditions (2) and (3) are then: every chosen integer is followed (clockwise) by at least $w$ non-chosen integers. Equivalently, such a $k$-choice can be described by a display of $k 1$ 's and $n-k 0$ 's in a circle with one of the $n$ entries capped. For example, the choice $\{2,5,9,12\}$ counted in $(13 \mid 4)_{2}$ is described by

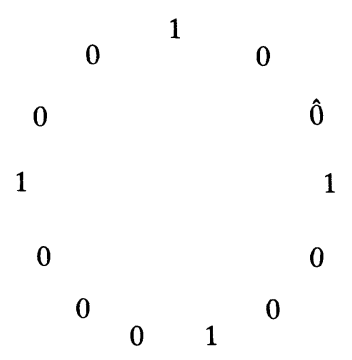

Received by the editors November 28, 1977 and, in revised form, April 26, 1979.

${ }^{1}$ Supported by NRC Grant \#A-3069.

${ }^{2}$ While visiting McGill University. 
(The cap tells you where to start!) It is well known [1, p. 222, problem 2] and easy to deduce [2, formula 17$]$ that

(4) $(n \mid k)_{w}= \begin{cases}\frac{n}{n-w k}\left(\begin{array}{c}n-w k \\ k\end{array}\right), & 0 \leq k \leq \frac{n}{w+1}, \quad(n, k) \neq(0,0), \\ 0, & 0 \leq \frac{n}{w+1}<k .\end{cases}$

Taking $(0 \mid 0)_{w}=w+1$ permits $(n \mid k)_{w}$ to be determined by the recurrence

$$
\begin{aligned}
& (n \mid k)_{w}=(n-1 \mid k)_{w}+(n-w-1 \mid k-1)_{w}, n \geq w+1, k \geq 1, \\
& (0 \mid 0)_{w}=w+1,(n \mid 0)_{w}=1 \text { for } n \geq 1,(n \mid k)_{w}=0 \text { for } 0 \leq n \leq w, k \geq 1 .
\end{aligned}
$$

Indeed, $(n-1 \mid k)_{w}$ counts the choices (1) (satisfying (2) and (3)) for which $x_{k}-x_{k-1}>w+1$, while $(n-w-1 \mid k-1)_{w}$ counts the choices for which $x_{k}-$ $x_{k-1}=w+1$.

When $w=0,(n \mid k)_{0}$ is simply the number of $k$-subsets of a set of size $n$, i.e.,

$$
(n \mid k)_{0}= \begin{cases}\left(\begin{array}{l}
n \\
k
\end{array}\right)=\frac{n !}{k !(n-k) !}, & 0 \leq k \leq n, \\
0, & 0 \leq n<k .\end{cases}
$$

The obvious identity

$$
\sum_{i=0}^{k}(m \mid i)_{0}(n \mid k-i)_{0}=(m+n \mid k)_{0}, \quad m, n, k \geq 0
$$

or equivalently,

$$
\sum_{i=0}^{k}\left(\begin{array}{c}
m \\
i
\end{array}\right)\left(\begin{array}{c}
n \\
k-i
\end{array}\right)=\left(\begin{array}{c}
m+n \\
k
\end{array}\right), \quad m, n, k \geq 0
$$

is the well known Vandermonde Convolution.

When $w=1$, we conveniently suppress the subscript 1 , so

$$
(n \mid k)=(n \mid k)_{1}=\left\{\begin{array}{cll}
2, & \text { if } \quad n=k=0, \\
\frac{n}{n-k}\left(\begin{array}{c}
n-k \\
k
\end{array}\right), & \text { if } & 0 \leq k \leq \frac{n}{2}, \quad(n, k) \neq(0,0), \\
0, & \text { if } & 0 \leq \frac{n}{2}<k .
\end{array}\right.
$$

\section{Defining}

$$
(m, n \mid k)_{w}=\sum_{i+j=k}(m \mid i)_{w}(n \mid j)_{w}
$$


the identity we establish is:

(6) $(m, n \mid k)=\left\{\begin{array}{lll}(m+n \mid k), & \text { if } 0 \leq k<m \leq n, \\ (m+n \mid k)+(-1)^{m}(n-m \mid k-m), & \text { if } 0 \leq m \leq n, m \leq k\end{array}\right.$

The combinatorial meaning of $(m, n \mid k)_{w}$ is this. It is the number of $k$-choices from $\{1,2, \ldots, m+n\}$ such that in the display of $\{1,2, \ldots, m\}$ in one circle and $\{m+1, \ldots, m+n\}$ in another circle each chosen integer is followed (in the circle it appears) by $w$ non-chosen integers.

After providing several proofs of (6), thus illustrating different techniques, we will describe an application to counting $3 \times n$ Latin rectangles, a generalization and its relation to an identity of Rothe ([9]; see [3] and [4]).

2 . When $w=1$, recurrence (5) is

$$
\begin{gathered}
(n \mid k)=(n-1 \mid k)+(n-2 \mid k-1), n \geq 2, k \geq 1, \\
(0 \mid 0)=2,(n \mid 0)=1 \text { for } n \geq 1,(n \mid k)=0 \text { for } n=0,1, k \geq 1,
\end{gathered}
$$

and this leads to the generating function which we "partial fraction":

$$
\sum_{n, k \geq 0}(n \mid k) x^{n} z^{k}=\frac{2-x}{1-x-x^{2} z}=\frac{1}{1-\alpha_{1} x}+\frac{1}{1-\alpha_{2} x}=\sum_{n \geq 0}\left(\alpha_{1}^{n}+\alpha_{2}^{n}\right) x^{n}
$$

where $\alpha_{1}, \alpha_{2}$ are power series in $z$ satisfying

$$
\alpha_{1}+\alpha_{2}=1, \quad \alpha_{1} \alpha_{2}=-z, \quad \alpha_{1}^{n}+\alpha_{2}^{n}=\sum_{k \geq 0}(n \mid k) z^{k}
$$

Now

$$
\begin{aligned}
\sum_{m, n, k \geq 0}(m, n \mid k) x^{m} y^{n} z^{k} & =\frac{2-x}{1-x-x^{2} z} \cdot \frac{2-y}{1-y-y^{2} z} \\
& =\sum_{m \geq 0}\left(\alpha_{1}^{m}+\alpha_{2}^{m}\right) x^{m} \sum_{n \geq 0}\left(\alpha_{1}^{n}+\alpha_{2}^{n}\right) y^{n}
\end{aligned}
$$

and equating coefficients of $x^{m} y^{n}$ yields for all $0 \leq m \leq n$

$$
\begin{aligned}
\sum_{k \geq 0}(m, n \mid k) z^{k} & =\left(\alpha_{1}^{m}+\alpha_{2}^{m}\right)\left(\alpha_{1}^{n}+\alpha_{2}^{n}\right) \\
& =\alpha_{1}^{m+n}+\alpha_{2}^{m+n}+\left(\alpha_{1} \alpha_{2}\right)^{m}\left(\alpha_{1}^{n-m}+\alpha_{2}^{n-m}\right) \\
& =\sum_{k \geq 0}(m+n \mid k) z^{k}+(-z)^{m} \sum_{k \geq 0}(n-m \mid k) z^{k} \\
& =\sum_{k \geq 0}(m+n \mid k) z^{k}+\sum_{k \geq m}(-1)^{m}(n-m \mid k-m) z^{k} .
\end{aligned}
$$

Equating coefficients of $z^{k}$ establishes identity (6). 
We can get by without partial fractioning. First note that

$$
\begin{aligned}
\sum_{m, n, k \geq 0}(m+n \mid k) x^{m} y^{n} z^{k} & =\sum_{r, k \geq 0}(r \mid k) z^{k} \sum_{m+n=r} x^{m} y^{n} \\
& =\sum_{r, k \geq 0}(r \mid k) z^{k} \frac{x^{r+1}-y^{r+1}}{x-y} \\
& =\frac{x}{x-y} \cdot \frac{2-x}{1-x-x^{2} z}+\frac{y}{y-x} \cdot \frac{2-y}{1-y-y^{2} z}
\end{aligned}
$$

Furthermore, letting

$$
a(m, n \mid k)=\left\{\begin{array}{c}
(-1)^{m}(n-m \mid k-m), \text { if } 0 \leq m \leq n, m \leq k, \\
(-1)^{n}(m-n \mid k-n), \quad \text { if } 0 \leq n<m, n \leq k, \\
0 \quad \text { if } k<\min (m, n),
\end{array}\right.
$$

a little manipulation yields

$$
\sum_{m, n, k \geq 0} a(m, n \mid k) x^{m} y^{n} z^{k}=\frac{1}{1+x y z}\left\{\frac{2-x}{1-x-x^{2} x}+\frac{2-y}{1-y-y^{2} z}-2\right\} .
$$

Identity (6) now follows because the sum of functions (8) and (9) is identically equal to function (7).

We proceed to outline an elementary proof of (6) which uses recurrences but not generating functions. Details are left to the reader. First note that

$$
\begin{aligned}
(m, n \mid k) & =(m-1, n \mid k)+(m-2, n \mid k-1), \quad m \geq 2, \quad n \geq 0, \quad k \geq 1, \\
& =(m, n-1 \mid k)+(m, n-2 \mid k-1), \quad m \geq 0, \quad n \geq 2, \quad k \geq 1,
\end{aligned}
$$

(cf. (5) with $w=1$ ). Next, taking

$$
g(m, n, k)=(m, n \mid k)-(m+n \mid k)+(m-1, n-1 \mid k-1)-(m+n-2 \mid k-1)
$$

for $m, n, k \geq 1$, it follows that

$$
\begin{aligned}
g(m, n, k)= & g(m-1, n-1, k)+g(m-1, n-2, k-1) \\
& +g(m-2, n-1, k-1)+g(m-2, n-2, k-2)
\end{aligned}
$$

for $m, n, k \geq 3$. Now

$$
g(m, n, k)=0 \quad \text { if } m, n, k \geq 0, m, n, k \text { not all } \geq 3,
$$

easily follows, and induction (using (10) and (11)) implies

or

$$
g(m, n, k)=0 \text { for } m, n, k \geq 1 \text {, }
$$

$$
(m, n \mid k)-(m+n \mid k)=-\{(m-1, n-1 \mid k-1)-(m+n-2 \mid k-1)\}
$$

for $m, n, k \geq 1$. Repeated application of (12) leads to (6). 
The last proof we now give of (6) is strictly combinatorial, by means of oneto-one correspondences. Let $S(m, n \mid k)$ denote the set of $k$-choices counted by $(m, n \mid k)$. Each such choice can be represented by a display

$$
\begin{aligned}
& \alpha_{1} \alpha_{2} \alpha_{3} \cdots \alpha_{m} \\
& \beta_{1} \beta_{2} \beta_{3} \cdots \beta_{m} \beta_{m+1} \cdots \beta_{n}, \quad 0 \leq m \leq n,
\end{aligned}
$$

of $k 1$ 's and $m+n-k 0$ 's. In each of the two rows no two 1's are adjacent, with $\alpha_{m}, \alpha_{1}$ and $\beta_{n}, \beta_{1}$ adjacent pairs. (These displays really should be in two circles, but that would make the typesetting difficult.)

The identity (6) is easy to prove when $0 \leq k<m \leq n$. In this case there is a $q$, $1 \leq q \leq m$, such that $\alpha_{q}=\beta_{q}=0$ while $\alpha_{i}, \beta_{i}$ are not both 0 when $i<q$. Consider the following mapping of a $k$-choice in $S(m, n \mid k)$ to a $k$-choice in $S(m+n \mid k)$ (the set counted by $(m+n \mid k))$ :

$$
\begin{aligned}
\alpha_{1} \alpha_{2} \cdots \alpha_{q-1} 0 \alpha_{q+1} & \cdots \alpha_{m} \\
\beta_{1} \beta_{2} \cdots \beta_{q-1} 0 \beta_{q+1} & \cdots \beta_{m} \cdots \beta_{n} \\
& \rightarrow \alpha_{1} \alpha_{2} \cdots \alpha_{q-1} 0 \beta_{q+1} \cdots \beta_{n} \beta_{1} \cdots \beta_{q-1} 0 \alpha_{q+1} \cdots \alpha_{m} .
\end{aligned}
$$

This is a one-to-one mapping from $S(m, n \mid k)$ to $S(m+n \mid k)$ when $0 \leq k<$ $m \leq n$. Furthermore, this map is onto $S(m+n \mid k)$. Indeed, if,

$$
\gamma_{1} \gamma_{2} \cdots \gamma_{m+n}
$$

is a sequence of $k 1$ 's and $m+n-k$ 0's representing a choice in $S(m+n \mid k)$ (so that no two 1's are adjacent, $\gamma_{m+n}$ and $\gamma_{1}$ being adjacent) then, because $k<m$, there is a $t, 1 \leq t \leq m$, such that $\gamma_{t}=\gamma_{t+n}=0$, while $\gamma_{i}, \gamma_{i+n}$ are not both 0 for $i<t$. Clearly the map takes the $k$-choice

$$
\begin{aligned}
& \gamma_{1} \gamma_{2} \cdots \gamma_{t-1} 0 \gamma_{n+t+1} \cdots \gamma_{n+m} \\
& \gamma_{1+n 2+n} \cdots \gamma_{t+n-1} 0 \gamma_{t+1} \cdots \gamma_{n}
\end{aligned}
$$

of $S(m, n \mid k)$ onto the $k$-choice (15), and hence $(m, n \mid k)=(m+n \mid k)$ if $0 \leq k<m \leq n$.

Several examples should make this correspondence clear. Thus for $k=3$, $m=4, n=6$ :

$$
\begin{aligned}
& \{5,7,9\} \text { or } \begin{array}{l}
0000 \\
101010
\end{array} \\
& \{1,6,9\} \text { or } \begin{array}{l}
1000010101000 \text { or }\{3,5,7\} \\
010010 \leftrightarrow 1000100100 \text { or }\{1,5,8\}
\end{array} \\
& \{2,5,7\} \text { or } \begin{array}{l}
0100 \\
101000 \leftrightarrow 0100001010 \text { or }\{2,7,9\}
\end{array}
\end{aligned}
$$

When $0 \leq m \leq n$ and $m \leq k$, the situation is slightly complicated. If $m=n \leq$ $k$, (6) is obviously correct. Hence we may take $m<n$. Consider first the case $m$ 
even. Partition the set $S(m, n \mid k)$ into three disjoint subsets $S_{1}, S_{2}, S_{3}$ as follows. $S_{1}$ consists of choices (13) for which there is a $q \in\{1,2, \ldots, m\}$ such that $\alpha_{q}=\beta_{q}=0$ while $\alpha_{i}, \beta_{i}$ are not both 0 for $i<q$. If there is no such $q$ then $\alpha_{i}+\beta_{i}=1,1 \leq i \leq m$, and the $k$-choice (13) belongs to $S_{2}$ if $\beta_{m}=\beta_{m+1}=0$, otherwise to $S_{3}$. The $k$-choices in $S_{1}$ are mapped by (14) to $k$-choices in $S(m+n \mid k)$; the $k$-choice (13) in $S_{2}$ is mapped to the $k$-choice

$$
\alpha_{1} \alpha_{2} \cdots \alpha_{m} \beta_{m+1} \beta_{m+2} \cdots \beta_{n} \beta_{1} \beta_{2} \cdots \beta_{m}
$$

in $S(m+n \mid k)$; the $k$-choice (13) in $S_{3}$ is mapped onto the $(k-m)$-choice

$$
\beta_{m+1} \beta_{m+2} \cdots \beta_{n}
$$

of $S(n-m \mid k-m)$. Illustrated below is a typical element of $S_{2}$ and two elements of $S_{3}$ and the mapping of each:

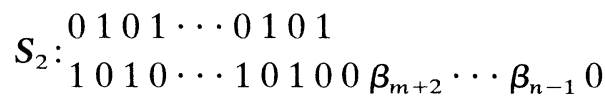

$$
\begin{aligned}
& \rightarrow 0101 \cdots 01010 \beta_{m+2} \cdots \beta_{n-1} 01010 \cdots 1010 \\
& \text { in } S(m+n \mid k) \text {; }
\end{aligned}
$$

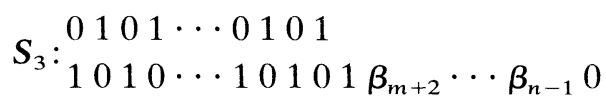

$$
\begin{aligned}
& \rightarrow 1 \beta_{m+2} \cdots \beta_{n-1} 0 \text { in } S(n-m \mid k-m) \text {; }
\end{aligned}
$$

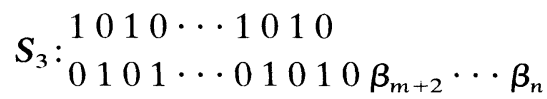

$$
\begin{aligned}
& \rightarrow 0 \beta_{m+2} \cdots \beta_{n} \text { in } S(n-m \mid k-m) .
\end{aligned}
$$

It is easy to see that $S_{1} \cup S_{2}$ is mapped one-to-one onto $S(m+n \mid k)$ while $S_{3}$ is mapped one-to-one onto $S(n-m \mid k-m)$, thus proving (6) for $0 \leq m \leq n$, $m<k, m$ even.

There remains the case $k \geq m, m$ odd. Now split $S(m, n \mid k)$ into two sets, $S_{1}$ as already described in the case of even $m$, and $S_{2}^{\prime}$ consisting of the $k$-choices (13) for which

$$
\alpha_{1} \alpha_{2} \cdots \alpha_{m}=01010 \cdots 010
$$

and

$$
\beta_{1} \beta_{2} \cdots \beta_{m}=10101 \cdots 101
$$

As before, the $k$-choices of $S_{1}$ are mapped by (14) to elements of $S(m+n \mid k)$ and the $k$ choice (13) in $S_{2}^{\prime}$ is mapped to the $k$-choice

$$
\alpha_{1} \alpha_{2} \cdots \alpha_{m} \beta_{m+1} \beta_{m+2} \cdots \beta_{n} \beta_{1} \cdots \beta_{m} .
$$

Note that $S_{1} \cup S_{2}^{\prime}$ is mapped one-to-one into $S(m+n \mid k)$, and the elements of 
$S(m+n \mid k)$ which are not images are the $k$-choices having one of the forms

$$
\begin{gathered}
01010 \cdots 01010 \gamma_{m+3} \cdots \gamma_{n-1} 010101 \cdots 101 \\
1010 \cdots 1010 \gamma_{m+2} \cdots \gamma_{n} 01010 \cdots 010 .
\end{gathered}
$$

The total number of these excluded $k$-choices in $S(m+n \mid k)$ is $(n-m \mid k-m)$, and the proof of (6) is complete.

There is an obvious generalization of (6) to any number of circles e.g.,

$$
\begin{aligned}
(10,4,17 \mid 15)= & (10+4+17 \mid 11)+(-1)^{10}(-10+4+17 \mid 15-10) \\
& +(-1)^{4}(10-4+17 \mid 15-4)+(-1)^{10+4}(-10-4+17 \mid 15-10-4) .
\end{aligned}
$$

3. Two permutations $a_{1}, \ldots, a_{n}$ and $b_{1}, \ldots, b_{n}$ of $\{1,2, \ldots, n\}$ are called discordant if $a_{i} \neq b_{i}, i=1, \ldots, n$. The Problème des Ménages asks for the number $u_{n}, n \geq 2$, of permutations discordant with the two permutations

$$
\begin{aligned}
& 123 \cdots n-1 n \\
& \text { n } 12 \cdots n-2 n-1 \text {. }
\end{aligned}
$$

Using $[i, j]$ to denote "the integer $i$ is in the $j$ th place", we seek permutations with none of the properties

$$
[1,1][1,2][2,2][2,3] \cdots[n-1, n-1][n-1, n][n, n][n, 1] .
$$

Since two of these properties are consistent if and only if they are not adjacent when the $2 n$ properties are arranged in a circle (so that $[1,1]$ follows $[n, 1])$, the Principles of Inclusion and Exclusion yields

$$
u_{n}=\sum_{k=0}^{n}(-1)^{k}(2 n \mid k)(n-k) !, \quad n \geq 2 .
$$

This is of course well known [5, p. 14].

Now let $u_{m, n}, 2 \leq m \leq n$, denote the number of permutations of $1,2, \ldots$, $m+n$ discordant with the two permutations

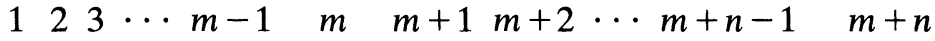

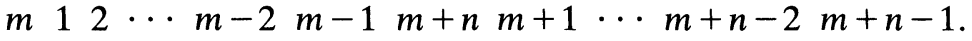

We seek permutations of degree $m+n$ having none of the properties

$$
[1,1][1,2][2,2][2,3] \cdots[m-1, m-1][m-1, m][m, m][m, 1]
$$

$[m+1, m+1][m+1, m+2][m+2, m+2][m+2, m+3]$

$$
\cdots[m+n-1, m+n][m+n, m+n][m+n, 1] \text {. }
$$

Clearly there are $(2 m, 2 n \mid k)$ ways of choosing, from these $2(m+n)$ properties, $k$ consistent ones. Hence

$$
u_{m, n}=\sum_{k=0}^{m+n}(-1)^{k}(2 m, 2 n \mid k)(m+n-k) !
$$


(and by (6))

$$
\begin{aligned}
& =\sum_{k=0}^{m+n}(-1)^{k}(2 m+2 n \mid k)(m+n-k) !+ \\
& +\sum_{k=2 m}^{m+n}(-1)^{k}(2 n-2 m \mid k-2 m)(n+m-k) ! \\
& =u_{m+n}+\sum_{i=0}^{n-m}(-1)^{i}(2 n-2 m \mid i)(n-m-i) ! \\
& =u_{m+n}+u_{n-m} .
\end{aligned}
$$

This formula also is well known ([1. p. 205], [8], [11, p. 15]), though our proof seems to be the first completely elementary one. It and its obvious generalization was used by Riordan to deduce a particularly elegant formula for the number of 3-line Latin rectangles [1, p. 205].

4. Although we have not been able to obtain a full generalization of (6) when $w \geq 1$ we can prove that

$$
(m, n \mid k)_{w}=(m+n \mid k)_{w}, \quad 0 \leq k<\frac{m}{w}, \frac{n}{w} .
$$

Let $S(m, n \mid k)_{w}$ denote the set of $k$-choices counted by $(m, n \mid k)_{w}$. Each such choice can be described by a display

$$
\begin{aligned}
& \alpha_{1} \alpha_{2} \cdots \alpha_{m} \\
& \beta_{1} \beta_{2} \cdots \beta_{m} \beta_{m+1} \cdots \beta_{n}, \quad 0 \leq m \leq n,
\end{aligned}
$$

of $k 1$ 's and $m+n-k$ 's and each 1 is followed by $w 0$ 's where we consider $\alpha_{m}, \beta_{n}$ to be followed by $\alpha_{1}, \beta_{1}$ respectively.

We consider two cases according to whether or not one of the $m-w+1$ "rectangles"

$$
R_{i}=\begin{aligned}
& \alpha_{i+1} \cdots \alpha_{i+w} \\
& \beta_{i+1} \cdots \beta_{i+w}
\end{aligned}, \quad 0 \leq i \leq m-w,
$$

has all entries 0 . We say such a rectangle is identically zero. If some $R_{i}$ is identically zero, choose $q$ so that $R_{q}$ is identically zero while for each $0 \leq i<q$ some entry in $R_{i}$ is 1 . Then

$$
\alpha_{1} \cdots \alpha_{q+w} \beta_{q+w+1} \cdots \beta_{n} \beta_{1} \cdots \beta_{q+w} \alpha_{q+w+1} \alpha_{m}
$$

is in $S(n+m \mid k)_{w}$.

If none of the rectangles $R_{i}, 0 \leq i \leq m-w$, is identically zero, then none of the disjoint rectangles $\boldsymbol{R}_{\ell_{w}}, 0 \leq \ell \leq[\mathrm{m} / \mathrm{w}]-1$, is identically zero and therefore each such $\boldsymbol{R}_{\ell_{w}}$ contains at least one 1 . Hence $[m / w]=k$ and each rectangle contains exactly one 1 . Thus $\alpha_{j}, \beta_{j}=0$ if $j>k w$. Because $k<(m / w)$ and no $\boldsymbol{R}_{i}$, $i=1, \ldots, m-w$, is identically zero, a simple count shows that one of the $w$ 
rectangles

$$
\begin{aligned}
& \alpha_{j+1} \cdots \alpha_{m} \alpha_{1} \cdots \alpha_{j-m+w} \\
& \beta_{j+1} \cdots \beta_{m} \beta_{1} \cdots \beta_{j-m+w}
\end{aligned}, \quad m-w \leq j<m,
$$

is identically zero, and we see that (17) looks like

$$
\begin{aligned}
& 00 \cdots 0 \alpha_{t+1} \alpha_{t+2} \cdots \alpha_{t+m-w} 00 \cdots 00 \\
& 00 \cdots 0 \beta_{t+1} \beta_{t+2} \cdots \beta_{t+m-w} 00 \cdots 00 \beta_{m+1} \cdots \beta_{n},
\end{aligned}
$$

for some $1 \leq t<w$. Thus

$$
\alpha_{1} \alpha_{2} \cdots \alpha_{m} \beta_{1} \cdots \beta_{n}
$$

is in $S(m+n \mid k)_{w}$.

Now we map display (17) to (18) in the first case and we map (17) to (19) in the second case. It is a simple matter to check that this mapping is one-to-one from $S(m, n \mid k)_{w}$ onto $S(m+n \mid k)_{w}$, and (16) is proved.

Several examples should make the above correspondence clear. Thus, for $w=3, m=10, n=12, k=3$ :

$\{11,15,19\}$ or 0000000000

$$
100010001000
$$$$
\leftrightarrow 0000100010001000000000 \text { or }\{5,9,13\} \text {; }
$$

$\{2,8,15\}$ or 0100000100 000010000000

$$
\leftrightarrow 0100000100000010000000 \text { or }\{2,8,15\} \text {; }
$$

$\{2,13,16\}$ or 0100000000

$$
001001000000
$$$$
\leftrightarrow 0100000000000010010000 \text { or }\{2,15,18\} .
$$

5. For integral $k \geq 0$ we define the polynomials of degree $k$ :

$$
\left(\begin{array}{l}
x \\
k
\end{array}\right)=\left\{\begin{array}{lll}
1, & \text { if } & k=0 \\
\frac{x(x-1) \cdots(x-k+1)}{k !}, & \text { if } & \mathrm{k}>0
\end{array}\right.
$$

and

$$
A_{k}(\alpha, \beta)=\frac{\alpha}{\alpha-k \beta}\left(\begin{array}{c}
\alpha-k \beta \\
k
\end{array}\right)
$$

The well known identity of Rothe [9] (see also [3], [4]) states:

$$
\sum_{i+j=k} A_{i}(\alpha, \beta) A_{j}(\gamma, \beta)=A_{k}(\alpha+\gamma, \beta) .
$$

It is an immediate consequence of (16). Indeed if $n, k, w$ are non-negative 
integers, $0 \leq k<(n / w)$, then by (4) $A_{k}(n, w)=(n \mid k)_{w}$. Thus the polynomial

$$
\sum_{i+j=k} A_{i}(\alpha, \beta) A_{j}(\gamma, \beta)-A_{k}(\alpha+\gamma, \beta)
$$

is 0 whenever $\alpha, \quad \gamma, \quad \beta$ are positive integers satisfying $1 \leq k<$ $\min (\alpha / \beta+1, \gamma / \beta+1)$, and this surely implies Rothe's identity when $k \geq 1$. For related material in a much more general setting, see [10]; for this and other identities proved by counting lattice paths see [6] and [7].

\section{REFERENCES}

1. J. Riordan, An Introduction to Combinatorial Analysis, Wiley, New York, 1958.

2. W. O. J. Moser and Morton Abramson, Enumeration of combinations with restricted differences and cospan, J. Comb. Theory 7 (1969), 162-170.

3. H. W. Gould, Some generalizations of Vandermonde's convolution, Amer. Math. Monthly 63 (1956), 84-91.

4. H. W. Gould, Final analysis of Vandermonde's convolution, Amer. Math. Monthly 64 (1957), 409-415.

5. M. Hall, Jr., Combinatorial Theory, Blaisdell, Toronto, 1967.

6. R. C. Lyness, Al Capone and the death ray, Math. Gazette 25 (1941), 283-287.

7. S. G. Mohanty, Lattice path counting and applications, Report No. 95, Dept. Math., McMaster University, 1977.

8. J. Touchard, Sur un problème de permutations, Comptes Rendus 198 (1934), 631-633.

9. H. A. Rothe, Dissertation, Leipzig, 1793.

10. G. N. Raney, Functional composition patterns and power series reversion, Trans. Amer. Math. Soc. 94 (1960), 441-451.

11. J. H. van Lint, Combinatorial Theory Seminar, Springer Lecture Notes No. 382, 1974.

Department of Mathematics

BURNSIDE HALL

MCGILl UNIVERSITY

Montreal, Que H3A2K6

MCGILL UNIVERSITY

NEW YORK UNIVERSITY

Courant Instrtute of Mathematical Sciences 